\title{
Childhood: a critical focus for 'primordial prevention' research
}

\author{
Valentin Fuster
}

The SPARK II Conference, held on 20 January 2003 , took up a process started by the National Heart, Lung, and Blood Institute, when a select group of scientists-the SPARK II Working Group-was assembled to assist in identifying important research opportunities that the institute should address over the next 5 years. The research schema comprised five areas of opportunity, including one on public health and another on health promotion. These topics are priorities for the research enterprise because there is an urgent need to understand and to check the increasing prevalence of cardiovascular disease.

Although cardiovascular disease typically occurs in middle age or later, risk factors are largely determined by behaviors learned in childhood, such as smoking and dietary habits. Longitudinal epidemiologic studies have shown that the measurement of traditional risk factors from childhood to adulthood can be useful in the prediction of subclinical cardiovascular disease in adults. Furthermore, post-mortem, pathologic and vessel-wall MRI analyses of children and adolescents who died in accidents have shown fatty and fibrous plaques in the coronary arteries; these early atherosclerotic lesions are frequently found in those whose risk factors included smoking, elevated plasma lipids, obesity and hypertension.

Children are increasingly adopting unhealthy lifestyles and developing the leading causes of heart disease and stroke-obesity, poor diet, inactivity and smoking - at an alarmingly early age. According to WHO, $60 \%$ of the world's children are inactive and consume too many calories. Globally, nearly $25 \%$ of all students smoke, having lit their first cigarette before the age of 10 years. The situation is worsened by passive smoke; almost half of the world's children live in the home of a smoker, and as a result have about a $25 \%$ increased risk of

\section{Although cardiovascular disease typically occurs in middle age or later, risk factors are largely determined by behaviors learned in childhood}

$V$ Fuster is the Editorin-Chief of Nature Clinical Practice Cardiovascular Medicine.

\section{Competing interests}

The author declared he has no competing interests.

www.nature.com/clinicalpractice doi:10.1038/ncpcardio0129 developing lung cancer and heart disease, and an $80 \%$ increased risk of stroke.

Longitudinal cardiovascular disease risk data from community-based investigations in children could provide an opportunity to study risk-factor modification for disease prevention. Such a community-guided strategy uses the public-health approach, in which the entire distribution of risk factors and risk is shifted toward lower levels through population-wide interventions and education. Such a worthy societal or community-driven strategy of health research and promotion has been referred to as primordial prevention.

The INTERHEART study, involving 52 countries and about 30,000 individuals, concluded that smoking, abdominal obesity, abnormal lipids, hypertension, diabetes, low consumption of fruits and vegetables, and lack of regular physical activity account for most of the myocardial infarction risk worldwide at all ages and in all regions. This and other studies also suggest that risk factors are mediated by shared family lifestyles (perhaps including some genetic background) rather than through independent pathways. If neither children nor parents perceive their health is being affected, however, it seems unlikely that they will seek health care or change their behavior. Therefore, to develop effective strategies to lessen or prevent such risks in childhood, we must first understand the societal, environmental (including family-related) and biological causes of the development of known risk factors.

I applaud the initiative of the National Institutes of Health in launching a study of a random sample across the US of 100,000 children to be assessed for health and environmental factors from the prenatal period to age 21 years. Indeed, childhood must be a critical focus for primordial prevention research. Unless the spread of risk factors is stemmed at this early age, the world faces an epidemic of cardiovascular disease. 